\title{
FOXRED1, encoding an FAD-dependent oxidoreductase complex-I-specific molecular chaperone, is mutated in infantile-onset mitochondrial encephalopathy
}

\author{
Elisa Fassone1,2, Andrew J. Duncan 1, Jan-Willem Taanman ${ }^{3}$, Alistair T. Pagnamenta ${ }^{4}$, \\ Michael I. Sadowski ${ }^{5}$, Tatjana Holand ${ }^{6}$, Waseem Qasim ${ }^{6}$, Paul Rutland ${ }^{1}$, Sarah E. Calvo ${ }^{7}$, \\ Vamsi K. Mootha ${ }^{7}$, Maria Bitner-Glindzicz ${ }^{1}$ and Shamima Rahman ${ }^{1,8,9, *}$ \\ ${ }^{1}$ Clinical and Molecular Genetics Unit, UCL Institute of Child Health, London WC1N 1EH, UK, ${ }^{2}$ Dino Ferrari Centre, \\ Department of Neurological Sciences, University of Milan, I.R.C.C.S. Foundation Ca' Granda, Ospedale Maggiore \\ Policlinico, 20122 Milan, Italy, ${ }^{3}$ Department of Clinical Neurosciences, Institute of Neurology, University College London, \\ London NW3 2PF, UK, ${ }^{4}$ Monaco Group, Wellcome Trust Centre for Human Genetics, Oxford OX3 7BN, UK, ${ }^{5}$ Division of \\ Mathematical Biology, National Institute for Medical Research, London NW7 1AA, UK, ${ }^{6}$ Molecular Immunology Unit, \\ Institute of Child Health, University College London, London WC1N 1EH, UK, ${ }^{7}$ Broad Institute of MIT and Harvard, \\ Cambridge, MA 02142, USA, ${ }^{8}$ MRC Centre for Neuromuscular Diseases, National Hospital for Neurology, Queen \\ Square, London WC1N 3BG, UK and ${ }^{9}$ Metabolic Unit, Great Ormond Street Hospital, London WC1N 3JH, UK
}

Received July 19, 2010; Revised August 27, 2010; Accepted September 16, 2010

Complex $I$ is the first and largest enzyme in the respiratory chain and is located in the inner mitochondrial membrane. Complex I deficiency is the most commonly reported mitochondrial disorder presenting in childhood, but the molecular basis of most cases remains elusive. We describe a patient with complex I deficiency caused by mutation of the molecular chaperone FOXRED1. A combined homozygosity mapping and bioinformatics approach in a consanguineous Iranian-Jewish pedigree led to the identification of a homozygous mutation in FOXRED1 in a child who presented with infantile-onset encephalomyopathy. Silencing of FOXRED1 in human fibroblasts resulted in reduced complex I steady-state levels and activity, while lentiviral-mediated FOXRED1 transgene expression rescued complex I deficiency in the patient fibroblasts. This FAD-dependent oxidoreductase, which has never previously been associated with human disease, is now shown to be a complex I-specific molecular chaperone. The discovery of the c.1054C >T; p.R352W mutation in the FOXRED1 gene is a further contribution towards resolving the complex puzzle of the genetic basis of human mitochondrial disease.

\section{INTRODUCTION}

Complex I (NADH : ubiquinone oxidoreductase, EC 1.6.5.3) is the largest enzyme in the inner mitochondrial membrane (IMM) and provides the entry point into the respiratory chain for electrons derived from fuel oxidation. However, despite its fundamental role in mitochondrial energy generation, complex I remains the least well understood of the respiratory chain complexes. Although the crystal structure has been elucidated (1), little is known about the function of many of the 45 subunits, and the factors necessary for and mechanisms of assembly of this macromolecular complex remain largely unknown.

Mitochondrial diseases are characterized by extreme clinical, biochemical and genetic heterogeneity (2). Isolated deficiency of complex I is the most commonly identified *To whom correspondence should be addressed at: Clinical and Molecular Genetics Unit, UCL Institute of Child Health, 30 Guilford Street, London
WC1N 1EH, UK. Tel: +44 2079052608; Fax: +44 2074046191; Email: s.rahman@ich.ucl.ac.uk

(C) The Author 2010. Published by Oxford University Press. All rights reserved.

For Permissions, please email: journals.permissions@oxfordjournals.org 
biochemical defect in childhood-onset mitochondrial disease (3). Only seven of the 45 different subunits of complex I are encoded by mitochondrial DNA (mtDNA), and mutations in these mitochondrial subunits account for $\sim 25 \%$ of complex I deficiency (4). Mutations in 12 of the 38 nuclear-encoded subunits (NDUFS1, MIM 157655; NDUFS2, MIM 602985; NDUFS3, MIM 603846; NDUFS4, MIM 602694; NDUFS6, MIM 603848; NDUFS7, MIM 601825; NDUFS8, MIM 602141; NDUFV1, MIM 161015; NDUFV2, MIM 600532; NDUFA1, MIM 300078; NDUFA2, MIM 602137; and NDUFA11, MIM 612638) have been implicated in a further $20 \%$ of complex I deficiency, most commonly presenting in childhood as fatal infantile lactic acidosis, Leigh syndrome, leukodystrophy or hypertrophic cardiomyopathy $(5,6)$.

More than half of complex I deficiency is believed to be caused by mutations in ancillary factors necessary for proper complex I assembly and functioning, but to date relatively few patients have been reported to have mitochondrial disease secondary to a mutation in a complex I assembly factor [NDUFAF1 (7), MIM 606934; NDUFAF2 (8), MIM 609653; NDUFAF3 (9), MIM 612911; NDUFAF4 (10), MIM 611776; C8ORF38 (11), MIM 612392; and C20ORF7 (12), MIM 612360]. Both mutations in structural complex I subunits and assembly factors reduce the amount of the fully assembled functional complex by affecting the rate of complex I assembly and/or its stability (5). Currently, treatment strategies for isolated complex I deficiency are lacking because of limited insights into its pathophysiology.

We now report a novel disorder affecting complex I activity and stability, caused by a homozygous mutation in the putative molecular chaperone FOXRED1, which we identified by a combined homozygosity mapping and bioinformatics approach.

\section{RESULTS}

\section{Biochemistry}

Spectrophotometric analysis of respiratory chain enzyme activities revealed severe isolated deficiency of complex I in skeletal muscle from the proband (7\% residual activity compared with the mean control, normalized for citrate synthase), with normal activities of other respiratory chain complexes (data not shown). There was a milder deficiency of complex I in patient fibroblasts, with $70 \%$ residual activity (data not shown). Immunoblot analysis of one-dimensional Blue-Native polyacrylamide gel electrophoresis (BN-PAGE) gels showed marked reduction of complex I holoenzyme in patient fibroblast mitochondria (Fig. 1).

\section{Homozygosity mapping and bioinformatics analysis}

Previous genetic analysis in this patient had excluded mtDNA mutations and mutations in seven structural subunits of complex I (NDUFS2, NDUFS3, NDUFS4, NDUFS6, NDUFS7, NDUFS8 and NDUFV1) as the cause of his complex I deficiency. We then used a homozygosity mapping approach to identify the responsible gene. Whole genome-wide SNP analysis revealed five candidate regions of homozygosity in the proband which were not shared with his five healthy siblings. These regions were further confirmed by microsatellite analysis, which allowed refinement of these five candidate intervals to a total of $50 \mathrm{Mb}$ (Supplementary Material, Table S1). These regions contained 338 genes. The longest region of homozygosity $(18.3 \mathrm{Mb})$ was observed on chromosome 6 , but did not contain any candidate genes implicated in complex I function. The third largest region of homozygosity $(9.2 \mathrm{Mb}$ on chromosome 18$)$ contained the only known structural subunit of complex I located within these homozygous intervals: NDUFV2 (MIM 600532; NM_021074).

The remaining 337 genes within the candidate regions were prioritized by bioinformatic analyses, including integration with the MitoCarta catalogue (11) and 11 genes encoding known or predicted mitochondrial proteins were selected for sequence analysis (Supplementary Material, Table S2).

\section{DNA sequence analysis}

No mutations were identified in NDUFV2. Only one possibly pathogenic mutation was identified in the other 11 genes sequenced: a homozygous c.1054C $>\mathrm{T}$ (p.R352W) mutation present in both predicted transcripts of FOXRED1 (NM_017547) on chromosome 11q24.2 (Fig. 2). Both parents of the proband were heterozygous for the mutation, as were two of his siblings. Three siblings were homozygous for the wild-type sequence (Fig. 3). The mutation was not found in 268 pan-ethnic healthy control alleles. Sequence analysis of 24 further patients with isolated complex I deficiency without a genetic diagnosis did not reveal any mutations in FOXRED1. The arginine at position 352 of the FOXRED1 protein is highly conserved through evolution (Fig. 4).

\section{Lentiviral-mediated complementation and silencing studies}

Mitochondrial complex I activity in patient fibroblasts was shown to be $\sim 70 \%$ of controls, relative to residual complex IV activity, using the mitochondrial dipstick assay kits (Fig. 5A), which was in agreement with the spectrophotometric assays. Expression of a synthetic FOXRED1 gene in patient fibroblasts, using a lentiviral vector, rescued the complex I activity to $\sim 90 \%$ of control levels (Fig. 5A), providing evidence that the $\mathrm{R} 352 \mathrm{~W}$ missense mutation in FOXRED1 was the cause of the observed complex I deficiency in the patient. However, transfection with an empty vector also increased complex I activity in patient fibroblasts slightly (Fig. 5A). The mild deficiency of complex I in patient fibroblasts probably explains why only a $10-20 \%$ increase in complex I activity was observed after lentiviral transfection with wild-type FOXRED1 cDNA compared with transfection with an empty vector.

Western blot analysis showed a reduced steady-state level of FOXRED1 in patient fibroblasts, which increased after lentiviral transduction with wild-type FOXRED1 cDNA (Fig. 5B). Western blot analysis also demonstrated a slight improvement in complex I amount (assessed with antiNDUFA9 antibody) in patient fibroblasts after transduction with wild-type FOXRED1 cDNA, compared with human control fibroblasts and patient fibroblasts transduced with an empty vector (Fig. 5B). These observations were confirmed by densitometric analysis of the western blots (Fig. 5B). 


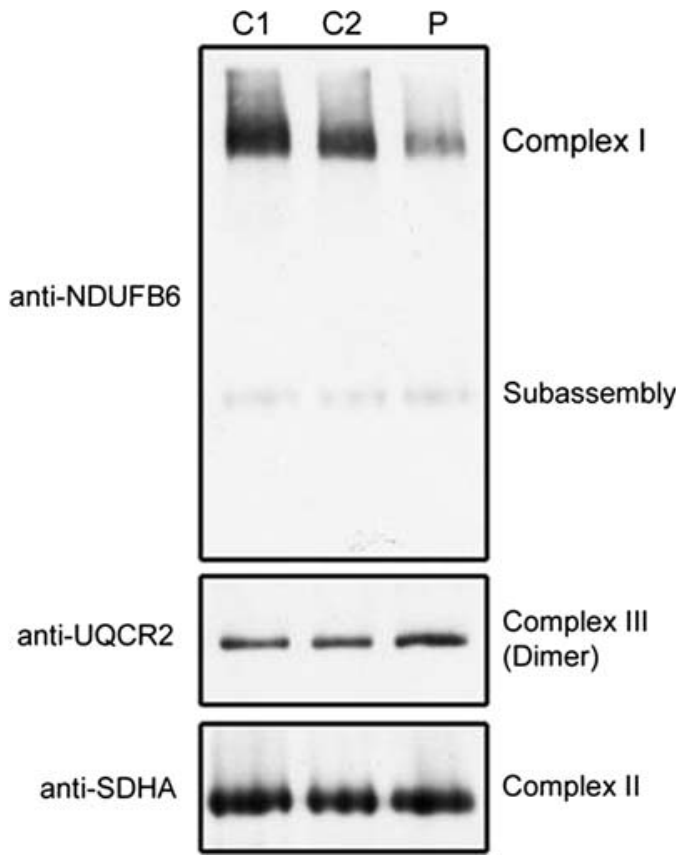

Figure 1. BN-PAGE of complex I holoenzyme. BN-PAGE shows that the complex I holoenzyme steady-state level is reduced in patient (P) fibroblasts compared with two healthy controls $(\mathrm{C} 1$ and $\mathrm{C} 2)$, probed with the antiNDUFB6 antibody. Probing for UQCR2 (anti-complex III) and SDHA (anti-complex II) demonstrated equal loading.

Stable knockdown of FOXRED1 expression via lentiviralmediated shRNAi-reduced complex I expression, assayed with anti-NDUFA9 antibody (Fig. 5B). Dipstick assay showed $\sim 40 \%$ residual complex I activity in FOXREDIsilenced fibroblasts compared with human control fibroblasts and scrambled fibroblasts (Fig. 5A), confirming that lack of FOXRED1 can cause complex I deficiency.

\section{Subcellular and submitochondrial localization}

Transfection of mammalian cells with a C-terminally labelled FOXRED1-YFP fusion plasmid demonstrated typical punctuate mitochondrial fluorescent staining, supporting mitochondrial localization of the protein (Fig. 6B). The western blot in Figure 7 shows that the anti-FOXRED1 antibody recognized a protein of the expected size $(\sim 53 \mathrm{kDa})$ in whole mitochondria, confirming mitochondrial localization of FOXRED1. A similar band of $\sim 53 \mathrm{kDa}$ is clearly visible in the mitochondrial inner membrane (IM) fraction and in the mitochondrial soluble fraction (i.e. mitochondrial inner membrane space (IMS) + matrix (M), indicated in Fig. 7 as 'Sol'). A similar slightly bigger polypeptide is detected in the IMS as well as in the matrix, indicating that FOXRED1 may enter the mitochondrial matrix as a precursor containing its mitochondrial import sequence, which is then cleaved to release the mature functional protein. Probing the same western blot with control antibodies anti-voltage-dependent anion channel (VDAC), anti-cytochrome $c$ and anti-MTCO2 (cytochrome $c$ oxidase subunit II) revealed the expected submitochondrial localizations for these proteins (outer membrane (OM), IMS and IM, respectively).
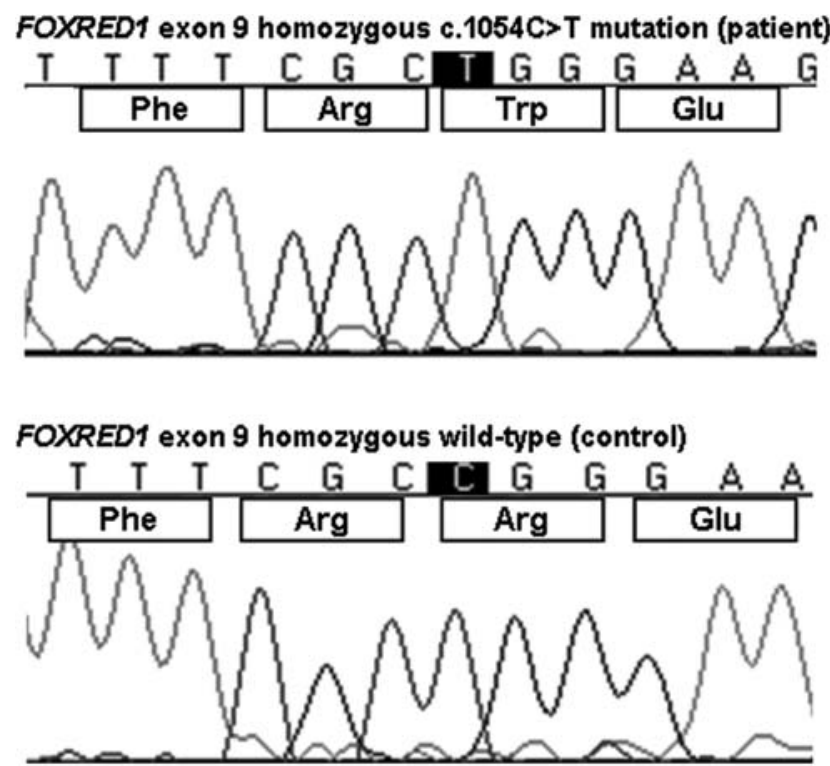

Figure 2. FOXRED1 exon 9 electropherogram. Electropherogram showing sequencing results for the FOXRED1 gene: the c. $1054 \mathrm{C}>\mathrm{T}$ mutation is homozygous in the patient (top panel) while the control sequence is wild-type homozygous (lower panel).

\section{FOXRED1 protein modelling}

The FOXRED1 (Uniprot AC: Q96CU9; ID: FXRD1_HUMAN) sequence was used to search the PDB using a profile generated versus the Swiss-Prot database (13) with four iterations of PSI-BLAST (14). PSI-BLAST was run with a profile inclusion score of 0.001 and default parameters otherwise. Several potential templates were identified; these were consistently related to the sarcosine oxidases. The top hit reported by the PSIBLAST run was $2 \mathrm{gb} 0$, a monomeric sarcosine oxidase from Bacillus spp. (15). The alignment was edited manually to ensure that the secondary structure of the observed template agreed with the prediction made by PSIPRED (16). The residue of interest, R352 in FOXRED1, aligns to F256 in the template, which is at the end of a short strand in the central sheet of the second domain in $2 \mathrm{gb} 0$ (Supplementary Material, Fig. S1). Protein modelling using the Bacillus monomeric sarcosine oxidase structure as a template suggested that mutation of arginine to tryptophan at position 352 could impinge on the FAD-binding site (Fig. 8).

\section{mRNA coexpression analysis}

Bioinformatics analysis using the mouse GNF1M tissue atlas showed a strong positive correlation between expression of FOXRED1 and expression of known complex I subunits (Supplementary Material, Fig. S2), implying a functional association. This correlation was stronger than for all previously identified assembly factors for complex I.

\section{DISCUSSION}

We used an integrative genomics approach, combining homozygosity mapping and bioinformatic analyses, to investigate a consanguineous Iranian-Jewish family in which the sixth child 


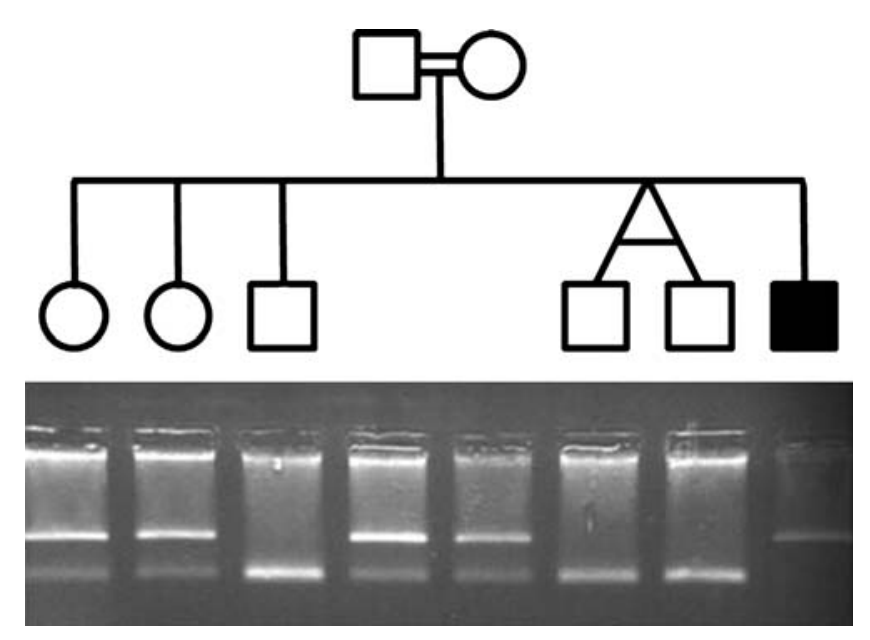

Figure 3. FOXRED1 mutation RFLP analysis. RFLP analysis shows segregation of the mutation with disease: none of the family members is homozygous for the c. $1054 \mathrm{C}>\mathrm{T}$ mutation except for the proband. The parents are heterozygous and unaffected siblings either heterozygous or homozygous for the wild-type sequence.

was affected by early-onset complex I deficient encephalomyopathy. Five candidate regions of homozygosity, unique to the proband and not shared with unaffected siblings, were identified by whole genome-wide SNP analysis. No mutations were identified in $N D U F V 2$, the only known structural subunit of complex I located within the homozygous intervals. Three hundred and thirty seven other genes located within the candidate regions were prioritized by bioinformatic analyses, and 11 genes encoding known or predicted mitochondrial proteins were sequenced.

The only pathogenic mutation identified in these 11 genes was a homozygous missense mutation c.1054C $>\mathrm{T}$ in FOXRED1. This mutation segregated with disease in the family; both parents and two siblings were heterozygous for the mutation, while the other three siblings were homozygous for the wild-type sequence. The mutation was not found in 268 pan-ethnic healthy control alleles. Western blot analysis showed a reduced steady-state level of FOXRED1 in patient fibroblasts and BN-PAGE analysis provided evidence of reduced amounts of complex I holoenzyme. Restoration of complex I activity after lentiviral transduction of patient fibroblasts with wild-type FOXRED1 cDNA confirmed that the FOXRED1 mutation is the cause of this patient's complex I deficiency.

FOXRED1 is a 486 amino acid protein, with an approximate mass of $53 \mathrm{kDa}$. It contains an FAD-dependent oxidoreductase domain, which may be involved in electron transfer. The mutation is predicted to cause the substitution of a highly conserved arginine residue by tryptophan (R352W). Our in silico modelling studies suggested that the mutation is likely to affect FOXRED1 protein structure. Mutation from arginine to the much larger tryptophan is likely to result in close proximity of the tryptophan sidechain to the FAD-binding site, thus interfering with FAD binding.

Mehrle et al. (17) have previously investigated subcellular localization of a large number of human proteins. Transfection of mammalian cells with a C-terminally labelled FOXRED1-
YFP fusion plasmid demonstrated typical punctuate mitochondrial fluorescent staining, supporting mitochondrial localization of the protein (Fig. 6B). Furthermore, an N-terminal CFP-FOXRED1 fusion protein failed to localize with mitochondria (Fig. 6A), suggesting that FOXRED1 has an Nterminal mitochondrial targeting sequence which was masked by the N-terminal fusion process (17). We performed in silico analysis using MitoProt II-v1.101 (18), which confirmed the presence of a putative $\mathrm{N}$-terminal mitochondrial localization sequence, spanning amino acids 1-23 (MIRRVLPHGMGRGLLTRRPGTRR). This sequence has a positive net charge of +1 and a predicted cleavage site at position 24; the probability of import into mitochondria was also very high at 0.9546 .

Our western blot data confirm mitochondrial localization of FOXRED1, with an expected band of $\sim 53 \mathrm{kDa}$ in the whole mitochondrial fraction (Fig. 7). In addition, a band slightly larger than $\sim 53 \mathrm{kDa}$ was observed in the IMS and the matrix. Based on this observation, we postulate that a FOXRED1 precursor containing its mitochondrial import sequence may be imported into mitochondria via a classical importation mechanism (such as the TOM/TIM machinery), and that the import sequence is subsequently cleaved within the matrix to release the mature protein (19). We also speculate that this mature protein then associates with the mitochondrial IM, near the respiratory chain supercomplexes and probably adjacent to the complex I holoenzyme, in order to exert its chaperone function.

FOXRED1 is not one of the known 45 subunits of complex I, as established by proteomics studies (20). However, the association of a homozygous FOXRED1 mutation with isolated complex I deficiency in our patient suggests that FOXRED1 must be a chaperone or other factor necessary for assembly, stability and/or correct functioning of complex I. Bioinformatics analysis using the mouse GNF1M tissue atlas showed coexpression of FOXRED1 with other complex I subunits, implying a functional association. A role for FOXRED1 in complex I activity is also supported by the absence of FOXRED1 orthologues in the yeast Saccharomyces cerevisiae, which has a functional respiratory chain but lacks the entire complex I holoenzyme, but the presence of FOXRED1 orthologues in higher order fungi such as Neurospora crassa, which do contain complex I. Further evidence indicating that FOXRED1 is needed for complex I function comes from a large-scale bioinformatic survey, which identified FOXRED1 as one of 19 putative complex I assembly factors (11). Finally, steady-state mRNA transcripts for FOXRED1 have been detected in 12 human tissues, implying that the protein is widely expressed, in common with all previously identified complex I assembly factors (Supplementary Material, Fig. S3).

Comparatively little is understood about the assembly process of complex I. The enzyme is L-shaped, containing membrane and matrix arms, and has three functional modules: the dehydrogenase $\mathrm{N}$ module (responsible for accepting electrons from NADH), the hydrogenase Q module (transfers electrons to coenzyme Q10) and the proton translocase $\mathrm{P}$ module (transfers protons across the IMM). The matrix arm contains the $\mathrm{N}$ and $\mathrm{Q}$ modules, while the $\mathrm{P}$ module is located in the membrane arm. Various 


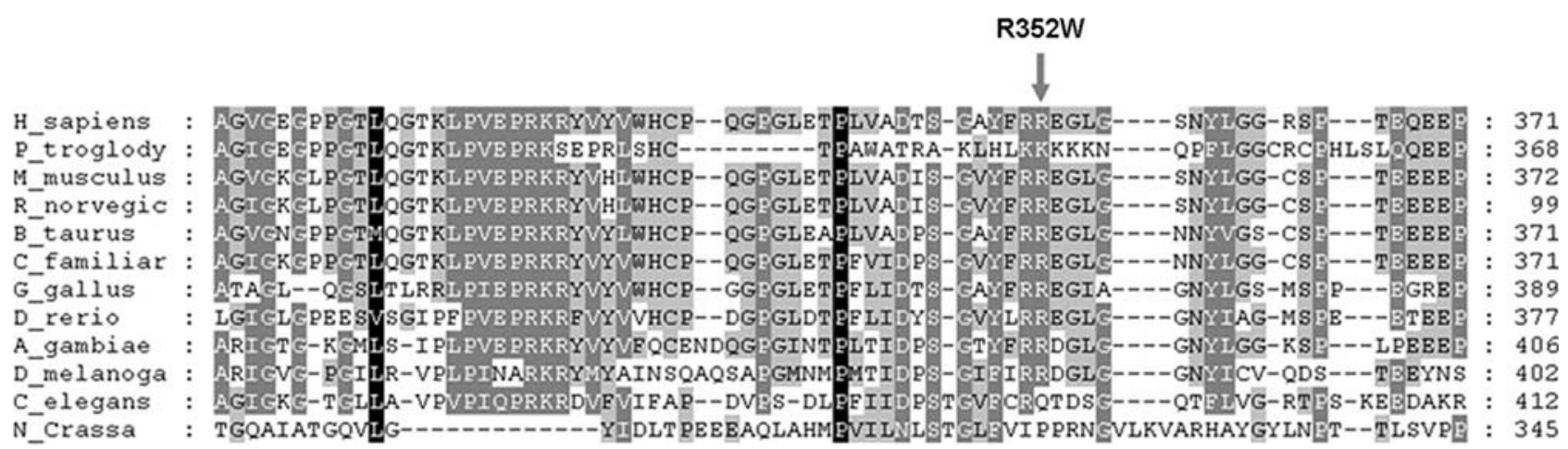

Figure 4. FOXRED1 protein evolutionary conservation. The FOXREDI protein has a well-conserved module in its structure across species. Within a part of this module, we found that the R352 residue is highly conserved across species, suggesting that the amino acid change R352W is likely to have a significant functional impact.

models have been suggested for complex I assembly, but the current consensus model proposes that the ND1 core subunit anchors an early Q subassembly to the IMM, then further subunits and/or subassemblies are added to both the $\mathrm{Q}$ and $\mathrm{P}$ modules and finally the $\mathrm{N}$ module is added to form the complex I holoenzyme (5). However, the precise order of subunit incorporation into the nascent enzyme is not known, nor the nature and number of additional factors required for integrity of the assembly process.

Human complex I deficiency has previously been linked to mutations in six complex I assembly factor genes (see above). Studies of assembly intermediates in fibroblasts from patients carrying mutations in these six genes have suggested that NDUFAF1 and C20ORF7 are involved early in complex I assembly, while NDUFAF2 appears to function at a later stage $(7,8,12)$. C20ORF7 appears to be necessary to form an early assembly intermediate of $\sim 400 \mathrm{kDa}$, which contains the ND1 subunit, while NDUFAF2 associates with a larger subassembly of $\sim 830 \mathrm{kDa}$. FOXRED1 constitutes a seventh putative assembly factor linked to human disease. There are also a number of putative assembly factors that have not yet been shown to cause human disease, including ECSIT (MIM 608388), PHB (MIM 176705), PTCD1 (NM_015545) and 17 further factors identified by Pagliarini et al. (11). It is likely that many more assembly factors of complex I will be identified, bearing in mind that the much smaller complex IV, which has only 13 subunits, requires more than 15 assembly factors for its assembly (21).

There does not appear to be any particular genotypephenotype correlation for the complex I assembly disorders, in common with many mitochondrial disorders (Table 1). Most patients presented with a progressive encephalopathy, frequently fulfilling the criteria for Leigh syndrome (22). Hypertrophic cardiomyopathy was observed in the patient with NDUFAF1 mutations, as well as our patient with FOXRED1 mutations. All patients had lactic acidaemia, except for those with NDUFAF2 mutations, in whom only CSF lactate levels were elevated. Severe isolated complex I deficiency was observed in skeletal muscle of all cases (residual activity $0-40 \%$ of controls), with normal activities of other respiratory chain enzyme complexes. There was a suggestion that NDUFAF2-related disease has a characteristic neuroradiological appearance, with a predilection for the mamillothalamic tracts, substantia nigra/medial lemniscus, medial longitudinal fasciculus and spinothalamic tracts, but this has not been found in all cases reported to date.

In the absence of clear genotype-phenotype correlations, the approach to a genetic diagnosis for patients with complex I deficiency remains empirical. The first-line genetic investigation (after screening for mtDNA rearrangements and common point mutations m.3243A $>\mathrm{G}$ and m. $8344 \mathrm{~A}>\mathrm{G}$ ) should be complete mitochondrial genome sequence analysis. Subsequent investigations will be directed at nuclear-encoded structural subunits and known assembly factors of complex I and/or homozygosity mapping in suitable families. However, this approach may not be successful in all cases, since an unknown number of complex I assembly factors remain to be identified, and since complex I deficiency may occasionally be secondary to another disease process, such as 3-hydroxyisobutyryl-CoA hydrolase deficiency (23). Taking into account the costs of sequencing the mitochondrial genome, 38 nuclear-encoded structural subunits and at least seven assembly factors, and the possibility of an underlying defect in a hitherto unrecognized gene, whole-exome sequence analysis using next-generation sequencing technologies may be a faster and cheaper route to diagnosis for many patients with isolated complex I deficiency in the future (24).

The localization of FOXRED1 within mitochondria, decrease in complex I activity upon silencing of its expression in wild-type fibroblasts and the complex I deficiency in patient muscle and fibroblasts, corrected by expression of wild-type FOXRED1 in patient fibroblasts, together with reduction of complex I holoenzyme assembly arising from a mutation in the coding sequence of FOXRED1, strongly support a bona fide role in complex I function, assembly and/or stability. Taken together, our results demonstrate that the R352W mutation in FOXRED1 causes an isolated complex I defect associated with infantile-onset encephalomyopathy, and that the FOXRED1 product is needed for fully efficient complex I activity in human cells.

Further studies are needed to incorporate known and unidentified complex I assembly factors into a more complete complex I assembly model. Identification of additional assembly factors remains a challenge, but should increase 


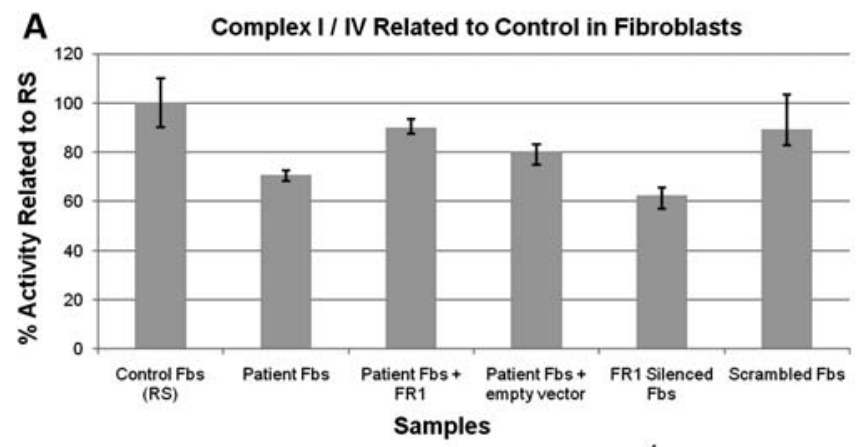

B

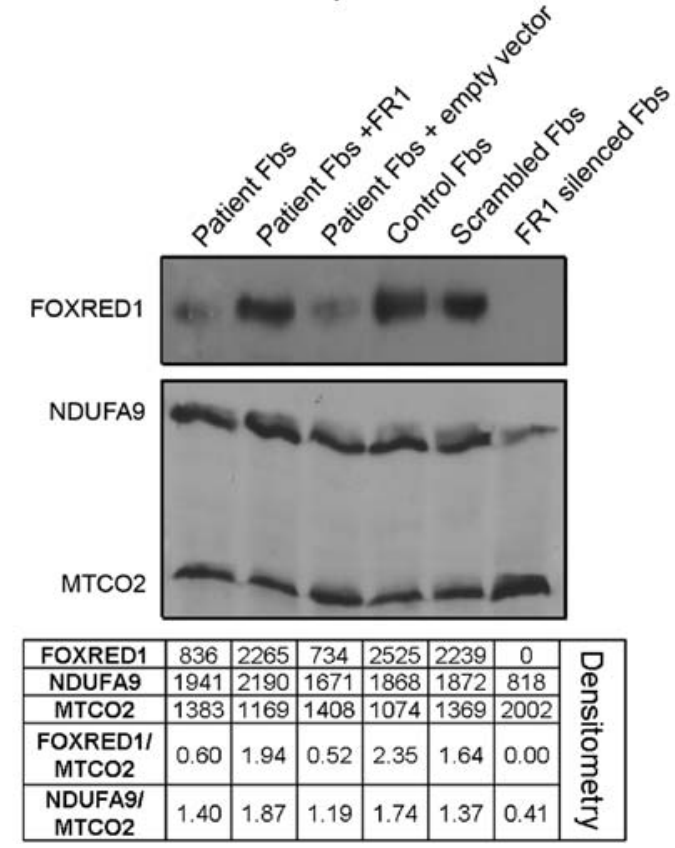

Figure 5. (A) Mitochondrial complex I/IV dipstick assay. Dipstick assay shows $\sim 70 \%$ residual complex I activity in patient fibroblasts (Fbs) which is recovered to $\sim 90 \%$ after complementation with synthetic wild-type FOXRED1 cDNA. A slight recovery is also seen after transfecting patient Fbs with an empty vector. FOXRED1-silenced fibroblasts show an $\sim 40 \%$ decrease in complex I activity compared with scrambled fibroblasts. Key: Fbs, fibroblasts; RS, reference sample; FR1, FOXRED1. (B) Western blot analysis. Western blot analysis shows a gain in FOXRED1 and NDUFA9 protein content in patient fibroblasts when complemented with FOXREDI wild-type cDNA, compared with patient fibroblasts transduced with an empty vector. FOXRED1-silenced fibroblasts show a reduction in FOXRED1 and NDUFA9 protein content compared with control and scrambled fibroblasts. Probing for MTCO2 (anti-complex IV) demonstrated equal loading. Densitometry measurements were carried out using Alpha Ease FC software (Alpha Innotech/Cell Biosciences, Santa Clara, CA, USA) and confirmed the observations above.

our understanding not only of complex I biogenesis but also of mitochondrial involvement in other common pathways or diseases, including neurodegeneration and cancer.

\section{MATERIALS AND METHODS}

\section{Patient}

The patient is the sixth child of healthy Iranian-Jewish first cousin parents. Two sisters and three brothers are unaffected. Pregnancy was uneventful, but soon after birth severe truncal
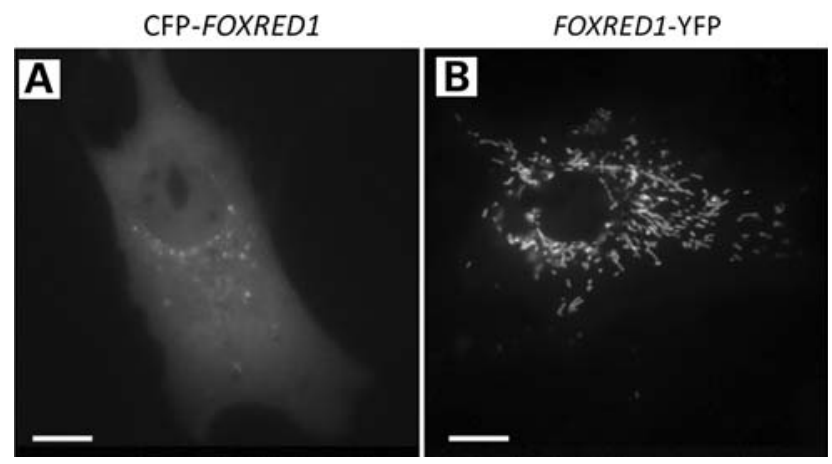

Figure 6. FOXRED1 mitochondrial localization. Figures taken from the LIFEdb database, clone ID: DKFZp586B1920. (A) Low-intensity non-specific cytoplasmic and nuclear staining of the N-terminally tagged FOXREDI protein. (B) High-intensity fluorescent staining of the C-terminally tagged FOXRED1 protein, with a characteristic punctate signal typical of a mitochondrially targeted protein. These images suggest that N-terminal tagging of the FOXRED1 protein interferes with the recognition of the mitochondrial targeting sequence. Bar, $10 \mu \mathrm{m}$.

hypotonia was noted. There have never been any voluntary movements. During the first year, he was extremely irritable with prolonged periods of inconsolable crying. Muscle biopsy was performed at 4 months of age because of persistent elevation of lactate in plasma $(6.8 \mathrm{~mm}$, normal $<2.1)$ and CSF $(4.3 \mathrm{~mm}$, normal $<1.8)$. Muscle histology was normal, but biochemical analysis revealed isolated deficiency of complex I in muscle mitochondria ( $7 \%$ of the control mean, normalized for citrate synthase activity), with normal activity of other respiratory chain enzyme complexes.

He developed progressive microcephaly, and brain MRI at 8 months revealed delayed myelination, ventricular dilatation and abnormal signal in the thalami and basal ganglia. Myoclonic jerks were present from 1 year but at 5 years responded to lamotrigine and clonazepam therapy. Eye movements have always been roving, with no evidence of visual function, and visual evoked potentials demonstrated absent cortical responses at 6 months of age. Bilateral optic atrophy was noted at 2 years. Hearing appears to be normal. Echocardiography revealed mild non-obstructive left ventricular hypertrophy. Poor feeding was present from birth, and from 5 years he has been fed by gastrostomy tube. He has had persistent hepatomegaly with normal liver transaminases and no signs of liver failure or cholestasis. He has had severe scoliosis from 5 years, treated with a corset. He was treated with riboflavin, uridine, creatine, lipoic acid, dichloroacetate and thiamine, without any obvious clinical response. He is currently 10 years old.

All samples were taken after informed patient/parental consent, and the study was approved by the local Ethics Committee.

\section{BN-PAGE analysis}

Assembled respiratory chain complexes were detected using BN-PAGE, performed as described previously (25). Samples were obtained from $1-2 \times 10^{6}$ cultured fibroblasts and mitochondrial-enriched pellets were produced by subcellular fractionation using differential centrifugation. The protease inhibitors pepstatin, leupeptin and phenylmethylsulphonyl 


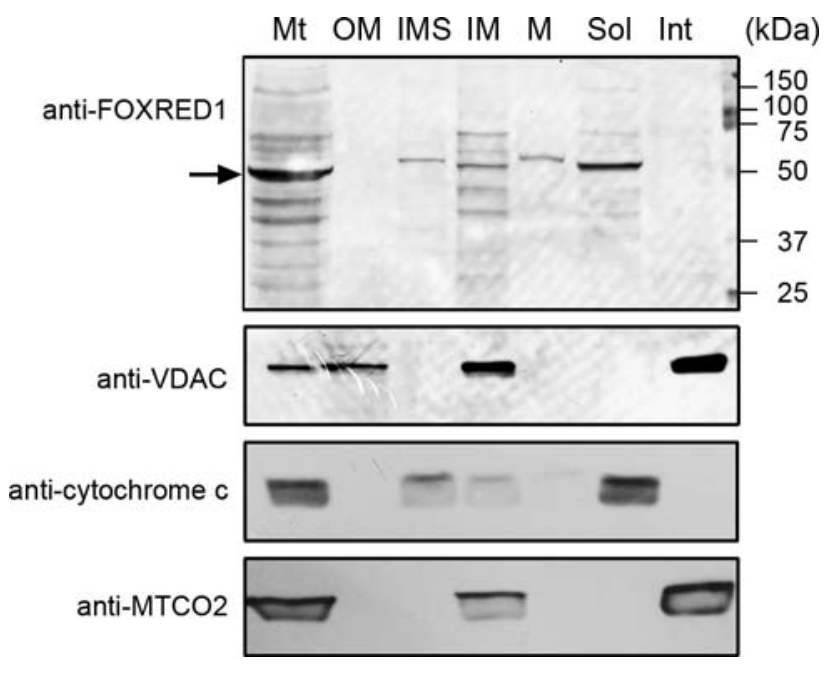

Figure 7. Submitochondrial fractionation. The $\sim 53 \mathrm{kDa}$ FOXRED1 protein (indicated by the arrow) shows a clear mitochondrial localization; more specifically, it is contained within the soluble fraction and associated with the IM. The slightly larger band detected in the IMS and the M may represent an immature polypeptide containing a mitochondrial targeting sequence, which is then cleaved to release the mature protein. The smaller mature protein then associates with the IM to achieve its fully functional state. Antibodies against mitochondrial proteins were used to test the purity of the fractions: anti-VDAC for the OM (and IM), anti-cytochrome $c$ for the IMS (also visible as associated with the IM) and anti-MTCO2 for the IM. Key: MT, whole mitochondria; OM, outer membrane; IMS, intermembrane space; M, matrix; SOL, soluble fraction; INT, integral proteins fraction.

fluoride were added to all homogenization, centrifugation and storage buffers. Mitochondrial protein fractions were solubilized with $n$-dodecyl-beta-D-maltoside, and $15-20 \mu l$ of the sample was loaded and run into $4-12 \%$ polyacrylamide gels, electrotransferred to Hybond-P membrane (GE healthcare) and probed with antibodies to subunit NDUFB6 (Mitosciences, OR, USA). The secondary antibody was goat anti-mouse conjugated to horseradish peroxidise (Dako). Equal loading was confirmed by reprobing blots with antiSDHA and anti-MTCO2 antibodies (Mitosciences).

\section{Genetic studies}

Whole genome-wide SNP genotyping was performed in the Iranian-Jewish proband and his unaffected siblings using the GeneChip Human Mapping 10K Xba Array (Affymetrix). We assumed an autosomal recessive model, and the output data were analysed assuming more than 30 consecutive homozygous SNPs as being significant. Five regions of significant homozygosity were observed in the patient but not his unaffected siblings, and these were further confirmed by microsatellite analysis. Microsatellites were selected using the Ensembl Human Genome Browser and PCR reactions were carried out using $5^{\prime}$ fluorescently labelled forward primers (primer sequences available upon request). After PCR amplification, the labelled DNA fragments were diluted 1/10-1/ 18 and then run together with the ET550-R size standard (final concentration $1 / 20$, Amersham Biosciences) at $10000 \mathrm{~V}$ for $75 \mathrm{~min}$ on an ABI MegaBACE ${ }^{\mathrm{TM}}$ cycle sequencer (Applied Biosystems), according to the manufacturer's

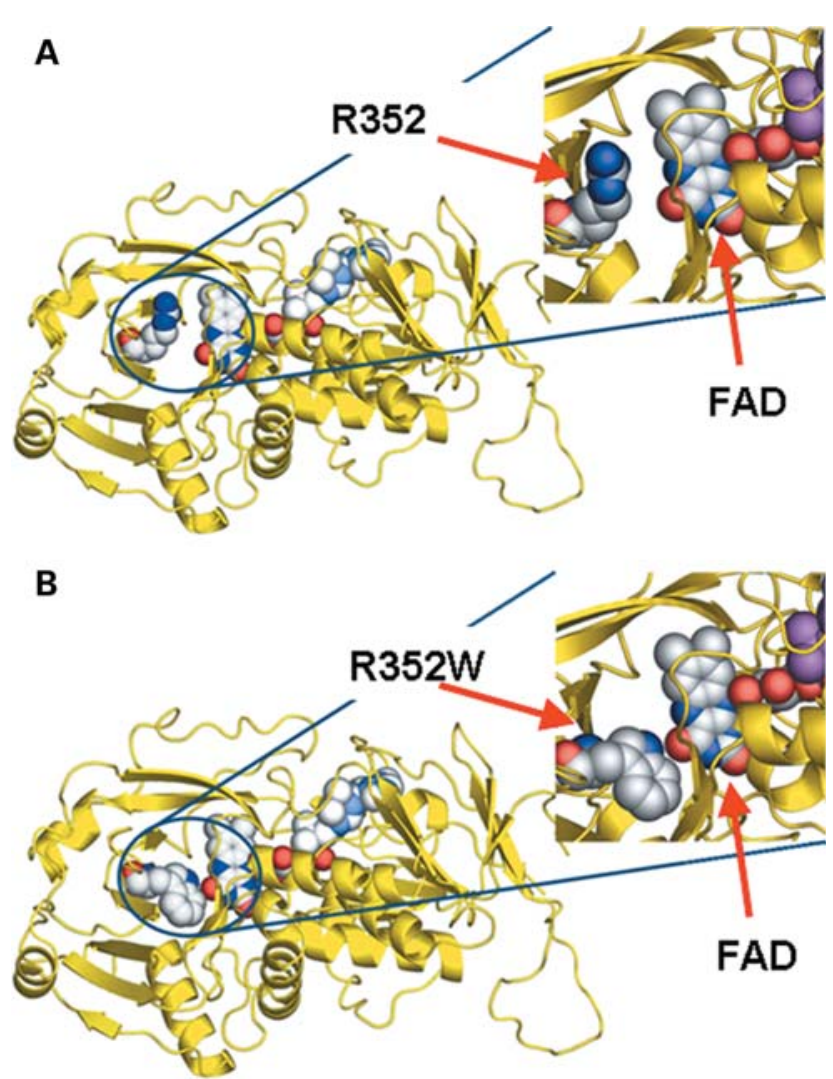

Figure 8. FOXRED1 protein modelling. Model of the FOXRED1 protein based on the Bacillus monomeric sarcosine oxidase structure (PDB code: $2 \mathrm{gb0}$ ). (A) The model with residue R352 and the FAD moiety shown in spacefilling representation. Inset is a close-up of the relation between R352 and the FAD. (B) The model of the R352W mutant. The observed mutation to tryptophan [R352W hypothetically visualized in (B)] could potentially place the mutant amino acid sidechain within contact distance of the FAD and interfere with binding.

instructions. Samples were denaturated at $95^{\circ} \mathrm{C}$ for $2 \mathrm{~min}$ and placed on ice before loading. Sample injection was at 3000 V for $45 \mathrm{~s}$. The data were analyzed using Genetic Profiler software v2.2 (Amersham Biosciences). The genes within the candidate regions were prioritized by bioinformatic analyses, including integration with the MitoCarta catalogue (11).

\section{DNA sequence analysis}

Eleven genes were sequenced by cycle sequencing (Big Dye v3.1 Terminator System) and analysis on MegaBACE ${ }^{\mathrm{TM}}$ (Supplementary Material, Table S2). Primer sequences for FOXRED1 are given in Supplementary Material, Table S3; primer sequences for the other 10 genes are available upon request. FOXRED1 exon 9 was amplified and sequenced using primers: forward: 5'-CCATGCTGTTTCTGCAGTT C-3'; reverse: 5'-GCCAAAAGCCTGATTGTTTC-3'. RFLP analysis was used to confirm the mutation: MspI (NewEngland Biolabs) cut the $245 \mathrm{bp}$ wild-type amplicon into two fragments of 121 and $124 \mathrm{bp}$. The mutation c.1054C $>\mathrm{T}$ abolished the restriction site, leaving the $245 \mathrm{bp}$ amplicon uncut. Two hundred and sixty eight pan-ethnic control alleles were screened for the c.1054C $>\mathrm{T}$ mutation in FOXRED1. The 
Table 1. Complex I assembly factors associated with disease

\begin{tabular}{|c|c|c|c|c|c|c|c|c|c|}
\hline Gene & Synonym(s) & $\begin{array}{l}\text { Number of } \\
\text { patients } \\
\text { (families) }\end{array}$ & Age at onset & Age at death & Clinical features & MRI features & $\begin{array}{l}\text { Maximum blood } \\
\text { lactate, } \mathrm{mm} \\
(\text { reference }<2 \text { ) }\end{array}$ & $\begin{array}{l}\text { Residual complex } \\
\text { I activity }\end{array}$ & References \\
\hline NDUFAF1 & CIA30 & $1(1)$ & $11 \mathrm{mo}$ & Alive at $20 \mathrm{y}$ & $\begin{array}{l}\text { Cardioencephalomyopathy } \\
\text { (HCM, WPW, cortical } \\
\text { visual failure, RP) }\end{array}$ & NS & 10 & $30 \% \mathrm{fb}^{\mathrm{a}}$ & (7) \\
\hline \multirow[t]{4}{*}{ NDUFAF2 } & \multirow[t]{4}{*}{$\begin{array}{l}\text { NDUFA12, } \\
\text { B17.2L, } \\
\text { Mimetin }\end{array}$} & $1(1)$ & $12 \mathrm{mo}$ & $13 y$ & $\begin{array}{l}\text { Progressive } \\
\text { encephalopathy }\end{array}$ & $\begin{array}{l}\text { Symmetrical lesions in } \\
\text { mamillothalamic tracts, } \\
\text { SN/ML, MLF, } \\
\text { spinothalamic tracts and } \\
\text { cerebellum }\end{array}$ & $\begin{array}{l}\text { Normal (CSF } \\
\quad \text { lactate } 4.2 \mathrm{~mm})\end{array}$ & $38 \% \mathrm{~m}^{\mathrm{a}}$ & (8) \\
\hline & & $2(2)$ & $20 \mathrm{mo} ; 8 \mathrm{mo}$ & $2 \mathrm{y} ; 21 \mathrm{mo}$ & $\begin{array}{l}\text { Progressive } \\
\text { encephalopathy }\end{array}$ & $\begin{array}{l}\text { Symmetrical lesions in } \\
\text { mamillothalamic tracts, } \\
\text { SN/ML, MLF and } \\
\text { spinothalamic tracts }\end{array}$ & $\begin{array}{l}\text { Normal (CSF } \\
\quad \text { lactate } 2.6 \mathrm{~mm})\end{array}$ & $\begin{array}{l}24-36 \% \mathrm{~m}^{\mathrm{a}} \\
53 \% \mathrm{fb}^{\mathrm{a}}\end{array}$ & (35) \\
\hline & & $1(1)$ & $3 \mathrm{mo}$ & $1 \mathrm{y}$ & LS & $\begin{array}{l}\text { Bilateral symmetrical lesions } \\
\text { in thalamus, cerebral } \\
\text { peduncles, brainstem and } \\
\text { spinal cord }\end{array}$ & 5 & $21-23 \% \mathrm{fb}^{\mathrm{b}}$ & (36) \\
\hline & & $1(1)$ & Ante-natal (IUGR) & $14 \mathrm{mo}$ & $\begin{array}{l}\text { Encephalomyopathy, } \\
\text { dysmorphism, } \\
\text { hepatopathy (NB } \\
\text { contiguous gene } \\
\text { deletion involving two } \\
\text { other genes ELOVL7 } \\
\text { and ERCC8) }\end{array}$ & Normal $(\mathrm{CT})$ & 3.4 & $45 \% \mathrm{fb}^{\mathrm{b}}$ & $(37)$ \\
\hline NDUFAF3 & C3orf60 & $5(3)$ & Neonate $(N=4)$ & $3-4 \mathrm{mo}$ & Lethal neonatal disease & Normal & 27 & $\begin{array}{l}26-40 \% \mathrm{~m}^{\mathrm{a}} \\
\quad 18-39 \% \mathrm{fb}^{\mathrm{a}}\end{array}$ & (9) \\
\hline NDUFAF4 & $\begin{array}{l}\text { C6orf66, } \\
\text { HRPAP20 }\end{array}$ & $5(1)$ & $\begin{array}{l}3 \mathrm{mo} \\
\text { Neonate }\end{array}$ & $\begin{array}{l}6 \mathrm{mo} \\
2-5 \mathrm{~d}(N=3) \\
\quad \text { alive at } 16 \mathrm{mo} \\
\quad \text { alive at } 7 \mathrm{y}\end{array}$ & $\begin{array}{l}\text { Myoclonic seizures } \\
\text { Infantile } \\
\quad \text { encephalomyopathy }\end{array}$ & $\begin{array}{l}\text { Diffuse leukomalacia } \\
\text { Severe atrophy of cerebrum, } \\
\text { cerebellum, basal ganglia, } \\
\text { pons and medulla }\end{array}$ & $\begin{array}{l}\text { NS } \\
38\end{array}$ & $\begin{array}{l}33 \% \mathrm{fb}^{\mathrm{a}} \\
0-21 \% \mathrm{~m}^{\mathrm{a}}\end{array}$ & $(10)$ \\
\hline C8ORF38 & & $2(1)$ & $7-10 \mathrm{mo}$ & $\begin{array}{l}34 \mathrm{mo} \text {; alive at } \\
22 \mathrm{mo}\end{array}$ & LS & LS & $\begin{array}{r}\text { Persistently } \\
\text { elevated }\end{array}$ & $\begin{array}{c}36 \% \mathrm{~m}^{\mathrm{a}} ; 20 \% \mathrm{l}^{\mathrm{a}} \\
14 \% \mathrm{fb}^{\mathrm{a}}\end{array}$ & (11) \\
\hline \multirow[t]{2}{*}{ C20ORF7 } & & $1(1)$ & Ante-natal (IUGR) & $7 \mathrm{~d}$ & $\begin{array}{l}\text { Dysmorphism, lethal } \\
\text { neonatal disease }\end{array}$ & $\begin{array}{l}\text { Agenesis of corpus callosum } \\
\text { (ultrasound) }\end{array}$ & 16.5 & $\begin{array}{l}25 \% \mathrm{~m}^{\mathrm{a}} ; 4 \% \mathrm{l}^{\mathrm{a}} \\
25 \% \mathrm{fb}^{\mathrm{a}}\end{array}$ & (12) \\
\hline & & $3(1)$ & $3 y$ & $\begin{array}{l}36 y \text {; alive at } 29 y \\
\text { alive at } 23 y\end{array}$ & LS & $\begin{array}{l}\text { Progressive atrophy and high } \\
\text { signal affecting caudate } \\
\text { nuclei and putamina }\end{array}$ & $\begin{array}{l}\text { Normal (CSF } \\
\text { lactate } 5 \mathrm{~mm})\end{array}$ & $36 \% \mathrm{~m} ; 6 \% \mathrm{pbmc}$ & $(38)$ \\
\hline FOXRED1 & & $1(1)$ & Neonate & Alive at $10 \mathrm{y}$ & & $\begin{array}{l}\text { Delayed myelination, } \\
\text { ventricular dilatation and } \\
\text { abnormal signal in thalami } \\
\text { and basal ganglia }\end{array}$ & 6.8 & $7 \% \mathrm{~m}^{\mathrm{a}}$ & This report \\
\hline
\end{tabular}

CT, computed tomography; d, days; fb, fibroblast; HCM, hypertrophic cardiomyopathy; IUGR, intrauterine growth retardation; 1, liver; LS, Leigh syndrome; m, muscle; ML, medial lemniscus; MLF, media longitudinal fasciculus; mo, months; MRI, magnetic resonance imaging; NS, not stated; pbmc, peripheral blood mononuclear cell; RP, pigmentary retinopathy; SN, substantia nigra; WPW, WolffParkinson-White syndrome; y, year.

${ }^{a}$ Percent normal control mean.

${ }^{b}$ Percent lowest control. 
FOXRED1 gene was also sequenced in 24 other patients with isolated complex I deficiency who did not have a genetic diagnosis.

\section{Western blot analysis}

Western blot analysis was performed as described elsewhere (25) on whole cell lysates (pre- and post-transduction), probing with anti-FOXRED1 antibody (Abnova, Taipei, Taiwan), and anti-NDUFA9 and anti-MTCO2 antibodies (Mitosciences). The secondary antibody was goat anti-mouse conjugated to horseradish peroxidise (Invitrogen). The analysis was performed at baseline and between days 15 and 20 after cell viral transduction. Densitometry measurements were carried out using Alpha Ease FC software (Alpha Innotech/Cell Biosciences, Santa Clara, CA, USA).

\section{Mitochondrial complex I and complex IV dipstick assay}

Activities of complexes I and IV were determined in whole cell lysates of patient and control fibroblasts with the Mitochondrial Dipstick Assay kit, performed according to the manufacturer's instructions (Mitosciences). Thirty micrograms of proteins were allowed to wick up laterally through the dipstick membrane, the dipsticks were transferred into the appropriate (complex I or complex IV) enzyme substrate buffer and enzyme activities were calculated by measuring the optical density of precipitating, colorimetric enzyme reaction products using the Image $\mathrm{J}^{\mathbb{\Omega}}$ program. Standard curves were constructed from multiple determinations of complex activities in cultured human fibroblast extracts; complex I $R^{2}$ 0.94-0.98 $(n=3)$ and complex IV $R^{2} 0.91-0.92(n=3)$. All measurements were done in triplicate and the results expressed as the complex I/complex IV activity ratio for each sample and compared with the fibroblast control range. All measurements were performed at baseline and between days 15 and 20 after cell viral transduction.

\section{Preparation of lentiviral vectors}

A synthetic codon-optimized FOXRED1 gene was designed to include a Kozak sequence (to increase translational initiation) and two STOP codons (to ensure efficient termination) and was generated by assembly of synthetic oligonucleotides (Genart, Regensburg). The gene was then subcloned into a lentiviral vector (26) and expression linked to eGFP through an internal ribosomal entry sequence. This vector utilizes a strong retroviral promoter element derived from the spleen focus-forming virus and a mutated woodchuck posttranslational regulatory element. Vector stocks were pseudotyped with the vesicular stomatitis virus envelope as described previously (27). Vector stocks of pGIPZ, a commercially available lentiviral vector system expressing puromycin/ eGFP markers and shRNAi against FOXREDl or scrambled shRNAi controls (Open Biosystems), were prepared in a similar manner for knock-down experiments. Vector stocks were titred by fluorescence-activated cell sorting (FACS) following dilutional transduction of a fixed number of 293T cells, and stored at $-80^{\circ} \mathrm{C}$ until required.

\section{Complementation studies}

Patient fibroblasts were cultured in DMEM and transduced with the lentiviral vector expressing eGFP alone or in combination with FOXRED1. Transduced cells were enriched for eGFP expression by FACS and expanded for functional studies.

\section{RNA interference studies}

Silencing the FOXRED1 gene in control fibroblasts was achieved by transducing the cells with the pGIPZ shRNAmir vector. Stably transduced clones were selected in $1 \mu \mathrm{g} / \mathrm{ml}$ puromycin (Gibco-Invitrogen). FOXRED1-silenced fibroblasts died within 20 days of culture following viral transduction.

\section{Submitochondrial fractionation}

Mitochondria from cultured HeLa S3 cells were isolated by differential centrifugation as reported previously (28). To subfractionate mitochondria, the swell-shrink procedure was used as described (29), except that the IMS was further purified by extraction in $10 \mathrm{~mm}$ Hepes. $\mathrm{NaOH}(\mathrm{pH} 7.4), 0.5 \mathrm{~mm}$ EDTA, $125 \mathrm{~mm}$ sucrose and $0.5 \%(\mathrm{v} / \mathrm{v})$ Triton X-100 on ice for $30 \mathrm{~min}$, followed by centrifugation at $160000 \mathrm{~g}, 4^{\circ} \mathrm{C}$ for $1 \mathrm{~h}$. To distinguish soluble and peripheral membrane proteins from integral membrane proteins, mitochondria were extracted with the alkaline carbonate method (30).

Purity of the subfractionated mitochondrial compartments was tested by probing with antibodies to detect a specific marker for each submitochondrial fraction: VDAC ( $\sim 30 \mathrm{kDa}$; Calbiochem, $86-173 / 016$, clone $31 \mathrm{HL})$ was used as an OM marker, cytochrome $c(\sim 12 \mathrm{kDa}$; BD Pharmingen, $556433)$ for the IMS and MTCO2 (26 kDa; Mitosciences, MS405, clone 12C4-F12) for the IM.

\section{Protein modelling}

PSI-BLAST (14) searches against the PDB resulted in several sarcosine oxidase hits. The model was generated using the Bacillus structure 2gb0 (15). Manual alignment editing was used to improve agreement with the secondary structure prediction generated by PSIPRED (16). The model was generated using MODELLER (31) with intermediate refinement (library schedule 1, 300 refinement cycles, three repeats, maximum PDF value 1e - 06); all non-protein atoms were inherited. Sidechain packing was re-optimized using SCWRL3 (32). All images were generated using PyMOL (33).

\section{mRNA coexpression analysis}

mRNA expression across 61 mouse tissues was performed using the GNF1M tissue atlas (34). The mouse tissue atlas contained probes corresponding to 909 distinct MitoCarta genes, including Foxred1. The mouse tissue atlas was used since probes targeting FOXRED1 were not present on the human GNF tissue atlas. Expression values were normalized across tissues based on the $Z$-score. The Pearson pairwise correlation was calculated for each gene pair, and this correlation matrix was clustered hierarchically using the $\mathrm{R}$ hclust function. 


\section{AUTHORS CONTRIBUTIONS}

S.R. was the overall study principal investigator who conceived the study and obtained financial support. S.R., E.F. and A.J.D. designed and oversaw the study, interpreted the results and drafted and synthesized the manuscript. J.-W.T. performed the BN-PAGE and western blot studies. A.T.P. performed the homozygosity mapping. M.I.S. modelled the human wild-type and mutated FOXRED1 protein. T.H. and W.Q. assisted in the lentiviral transduction. P.R. helped with acquisition and analysis of sequence data. S.E.C. and V.K.M. investigated the bioinformatics profiles of candidate genes within homozygous regions. M.B.-G. co-supervised the homozygosity mapping and revised the manuscript.

\section{SUPPLEMENTARY MATERIAL}

Supplementary Material is available at $H M G$ online.

\section{ACKNOWLEDGEMENTS}

We thank Dr Stefan Wiemann for permission to publish the mitochondrial localization images of FOXRED1, and Dr Amanda Heslegrave and Kerra Pearce for technical assistance. We thank Professor Robert Taylor for providing DNA samples from additional patients with complex I deficiency.

Conflict of Interest statement. None declared.

\section{FUNDING}

This work was supported by the UK Medical Research Council (G0200335 to S.R. and career development fellowship to M.I.S.), the Child Health Research Appeal Trust, Jeans 4 Genes, Climb and Leukaemia and Lymphoma research.

\section{REFERENCES}

1. Efremov, R.G., Baradaran, R. and Sazanov, L.A. (2010) The architecture of respiratory complex I. Nature, 465, 441-445.

2. Rahman, S. and Hanna, M.G. (2009) Diagnosis and therapy in neuromuscular disorders: diagnosis and new treatments in mitochondrial diseases. J. Neurol. Neurosurg. Psychiatry, 80, 943-953.

3. Kirby, D.M., Crawford, M., Cleary, M.A., Dahl, H.H., Dennett, X. and Thorburn, D.R. (1999) Respiratory chain complex I deficiency: an underdiagnosed energy generation disorder. Neurology, 52, 1255-1264.

4. Thorburn, D.R., Sugiana, C., Salemi, R., Kirby, D.M., Worgan, L., Ohtake, A. and Ryan, M.T. (2004) Biochemical and molecular diagnosis of mitochondrial respiratory chain disorders. Biochim. Biophys. Acta, 1659, 121-128

5. Lazarou, M., Thorburn, D.R., Ryan, M.T. and McKenzie, M. (2009) Assembly of mitochondrial complex I and defects in disease. Biochim. Biophys. Acta, 1793, 78-88.

6. Hoefs, S.J., Dieteren, C.E., Distelmaier, F., Janssen, R.J., Epplen, A., Swarts, H.G., Forkink, M., Rodenburg, R.J., Nijtmans, L.G., Willems, P.H. et al. (2008) NDUFA2 complex I mutation leads to Leigh disease. Am. J. Hum. Genet., 82, 1306-1315.

7. Dunning, C.J., McKenzie, M., Sugiana, C., Lazarou, M., Silke, J., Connelly, A., Fletcher, J.M., Kirby, D.M., Thorburn, D.R. and Ryan, M.T. (2007) Human CIA30 is involved in the early assembly of mitochondrial complex I and mutations in its gene cause disease. EMBO J., 26, 32273237 .
8. Ogilvie, I., Kennaway, N.G. and Shoubridge, E.A. (2005) A molecular chaperone for mitochondrial complex I assembly is mutated in a progressive encephalopathy. J. Clin. Invest, 115, 2784-2792.

9. Saada, A., Vogel, R.O., Hoefs, S.J., van den Brand, M.A., Wessels, H.J., Willems, P.H., Venselaar, H., Shaag, A., Barghuti, F., Reish, O. et al. (2009) Mutations in NDUFAF3 (C3ORF60), encoding an NDUFAF4 (C6ORF66)-interacting complex I assembly protein, cause fatal neonatal mitochondrial disease. Am. J. Hum. Genet., 84, 718-727.

10. Saada, A., Edvardson, S., Rapoport, M., Shaag, A., Amry, K., Miller, C. Lorberboum-Galski, H. and Elpeleg, O. (2008) C6ORF66 is an assembly factor of mitochondrial complex I. Am. J. Hum. Genet., 82, 32-38.

11. Pagliarini, D.J., Calvo, S.E., Chang, B., Sheth, S.A., Vafai, S.B., Ong, S.E., Walford, G.A., Sugiana, C., Boneh, A., Chen, W.K. et al. (2008) A mitochondrial protein compendium elucidates complex I disease biology. Cell, 134, 112-123.

12. Sugiana, C., Pagliarini, D.J., McKenzie, M., Kirby, D.M., Salemi, R., Abu-Amero, K.K., Dahl, H.H., Hutchison, W.M., Vascotto, K.A., Smith, S.M. et al. (2008) Mutation of C20orf7 disrupts complex I assembly and causes lethal neonatal mitochondrial disease. Am. J. Hum. Genet., 83, 468-478.

13. Boeckmann, B., Bairoch, A., Apweiler, R., Blatter, M.C., Estreicher, A., Gasteiger, E., Martin, M.J., Michoud, K., O’Donovan, C., Phan, I. et al. (2003) The SWISS-PROT protein knowledgebase and its supplement TrEMBL in 2003. Nucleic Acids Res., 31, 365-370.

14. Altschul, S.F., Madden, T.L., Schaffer, A.A., Zhang, J., Zhang, Z., Miller, W. and Lipman, D.J. (1997) Gapped BLAST and PSI-BLAST: a new generation of protein database search programs. Nucleic Acids Res., 25, 3389-3402.

15. Trickey, P., Wagner, M.A., Jorns, M.S. and Mathews, F.S. (1999) Monomeric sarcosine oxidase: structure of a covalently flavinylated amine oxidizing enzyme. Structure, 7, 331-345.

16. Jones, D.T. (1999) Protein secondary structure prediction based on position-specific scoring matrices. J. Mol. Biol., 292, 195-202.

17. Mehrle, A., Rosenfelder, H., Schupp, I., del, V.C., Arlt, D., Hahne, F., Bechtel, S., Simpson, J., Hofmann, O., Hide, W. et al. (2006) The LIFEdb database in 2006. Nucleic Acids Res., 34, D415-D418.

18. Claros, M.G. and Vincens, P. (1996) Computational method to predict mitochondrially imported proteins and their targeting sequences. Eur. J. Biochem., 241, 779-786.

19. Schmidt, O., Pfanner, N. and Meisinger, C. (2010) Mitochondrial protein import: from proteomics to functional mechanisms. Nat. Rev. Mol. Cell Biol., 11, 655-667.

20. Carroll, J., Fearnley, I.M., Skehel, J.M., Shannon, R.J., Hirst, J. and Walker, J.E. (2006) Bovine complex I is a complex of 45 different subunits. J. Biol. Chem., 281, 32724-32727.

21. Fontanesi, F., Soto, I.C., Horn, D. and Barrientos, A. (2006) Assembly of mitochondrial cytochrome c-oxidase, a complicated and highly regulated cellular process. Am. J. Physiol. Cell Physiol., 291, C1129-C1147.

22. Rahman, S., Blok, R.B., Dahl, H.H., Danks, D.M., Kirby, D.M., Chow, C.W., Christodoulou, J. and Thorburn, D.R. (1996) Leigh syndrome: clinical features and biochemical and DNA abnormalities. Ann. Neurol., 39, 343-351.

23. Loupatty, F.J., Clayton, P.T., Ruiter, J.P., Ofman, R., Ijlst, L., Brown, G.K., Thorburn, D.R., Harris, R.A., Duran, M., Desousa, C. et al. (2007) Mutations in the gene encoding 3-hydroxyisobutyryl-CoA hydrolase results in progressive infantile neurodegeneration. Am. J. Hum. Genet., 80, 195-199.

24. Ng, S.B., Buckingham, K.J., Lee, C., Bigham, A.W., Tabor, H.K., Dent, K.M., Huff, C.D., Shannon, P.T., Jabs, E.W., Nickerson, D.A. et al. (2010) Exome sequencing identifies the cause of a Mendelian disorder. Nat. Genet., 42, 30-35.

25. Williams, S.L., Valnot, I., Rustin, P. and Taanman, J.W. (2004) Cytochrome c oxidase subassemblies in fibroblast cultures from patients carrying mutations in COX10, SCO1, or SURF1. J. Biol. Chem., 279, $7462-7469$.

26. Qasim, W., Mackey, T., Sinclair, J., Chatziandreou, I., Kinnon, C., Thrasher, A.J. and Gaspar, H.B. (2007) Lentiviral vectors for T-cell suicide gene therapy: preservation of T-cell effector function after cytokine-mediated transduction. Mol. Ther., 15, 355-360.

27. Demaison, C., Parsley, K., Brouns, G., Scherr, M., Battmer, K., Kinnon, C., Grez, M. and Thrasher, A.J. (2002) High-level transduction and gene expression in hematopoietic repopulating cells using a human immunodeficiency [correction of immunodeficiency] virus type 1-based 
lentiviral vector containing an internal spleen focus forming virus promoter. Hum. Gene Ther., 13, 803-813.

28. Rickwood, D., Wilson, M.T. and Darley-Usmar, V.M. (1987) In Darley-Usmar, V.M., Rickwood, D. and Wilson, M.T. (eds), Mitochondria: a practical approach, IRL Press Ltd, p. 6.

29. Zhang, L., Shimoji, M., Thomas, B., Moore, D.J., Yu, S.W., Marupudi, N.I., Torp, R., Torgner, I.A., Ottersen, O.P., Dawson, T.M. and Dawson, V.L. (2005) Mitochondrial localization of the Parkinson's disease related protein DJ-1: implications for pathogenesis. Hum. Mol. Genet., 14, $2063-$ 2073.

30. Fujiki, Y., Hubbard, A.L., Fowler, S. and Lazarow, P.B. (1982) Isolation of intracellular membranes by means of sodium carbonate treatment: application to endoplasmic reticulum. J. Cell Biol., 93, 97-102.

31. Sali, A. and Blundell, T.L. (1993) Comparative protein modelling by satisfaction of spatial restraints. J. Mol. Biol., 234, 779-815.

32. Canutescu, A.A., Shelenkov, A.A. and Dunbrack, R.L. Jr. (2003) A graph-theory algorithm for rapid protein side-chain prediction. Protein Sci., 12, 2001-2014.

33. DeLano, W.L. (2002) The PyMOL Molecular Graphics System. DeLano Scientific, San Carlos, CA, USA. http://www.pymol.org. Accessed November 2007.
34. Su, A.I., Wiltshire, T., Batalov, S., Lapp, H., Ching, K.A., Block, D., Zhang, J., Soden, R., Hayakawa, M., Kreiman, G. et al. (2004) A gene atlas of the mouse and human protein-encoding transcriptomes. Proc. Natl Acad. Sci. USA, 101, 6062-6067.

35. Barghuti, F., Elian, K., Gomori, J.M., Shaag, A., Edvardson, S., Saada, A. and Elpeleg, O. (2008) The unique neuroradiology of complex I deficiency due to NDUFA12L defect. Mol. Genet. Metab., 94, 78-82.

36. Hoefs, S.J., Dieteren, C.E., Rodenburg, R.J., Naess, K., Bruhn, H., Wibom, R., Wagena, E., Willems, P.H., Smeitink, J.A., Nijtmans, L.G. et al. (2009) Baculovirus complementation restores a novel NDUFAF2 mutation causing complex I deficiency. Hum. Mutat., 30, E728-E736.

37. Janssen, R.J., Distelmaier, F., Smeets, R., Wijnhoven, T., Ostergaard, E., Jaspers, N.G., Raams, A., Kemp, S., Rodenburg, R.J., Willems, P.H. et al. (2009) Contiguous gene deletion of ELOVL7, ERCC8 and NDUFAF2 in a patient with a fatal multisystem disorder. Hum. Mol. Genet., 18, $3365-$ 3374.

38. Gerards, M., Sluiter, W., van den Bosch, B.J., de, W.E., Calis, C.M., Frentzen, M., Akbari, H., Schoonderwoerd, K., Scholte, H.R., Jongbloed, R.J. et al. (2010) Defective complex I assembly due to C20orf7 mutations as a new cause of Leigh syndrome. J. Med. Genet., 47, 507-512. 